PROCEEDINGS OF THE

AMERICAN MATHEMATICAL SOCIETY

Volume 138, Number 10, October 2010, Pages 3569-3583

S 0002-9939(10)10355-4

Article electronically published on April 7, 2010

\title{
CONNECTEDNESS OF THE ARNOLD TONGUES FOR DOUBLE STANDARD MAPS
}

\author{
ALEXANDRE DEZOTTI
}

(Communicated by Bryna Kra)

\begin{abstract}
We show that Arnold tongues for the family of double standard maps

$$
f_{a, b}(x)=2 x+a-(b / \pi) \sin (2 \pi x)
$$

are connected. This proof is accomplished in the complex domain by means of quasiconformal techniques and depends partly upon the fact that the complexification of $f_{a, b}$, has only one critical orbit taking symmetry into account.
\end{abstract}

\section{INTRODUCTION}

Our object is to study the family of mappings from the circle to itself, which we will call double standard maps, described below. The starting point is the family of real-analytic mappings $F_{a, b}: \mathbb{R} \rightarrow \mathbb{R}$ given by

$$
X \mapsto F_{a, b}(X)=2 X+a-\frac{b}{\pi} \sin (2 \pi X),
$$

with $(a, b) \in \mathbb{R} \times[0,1]$. These functions descend to mappings $f_{a, b}: \mathbb{T}^{1} \rightarrow \mathbb{T}^{1}$, where $\mathbb{T}^{1}=\mathbb{R} / \mathbb{Z}$. We call this the double standard family $\left(f_{a, b}\right)_{a \in \mathbb{T}^{1}, b \in[0,1]}$. This is a family of degree 2 mappings of the circle which has been recently studied by Ana Rodrigues and Michał Misiurewicz [4], [5].

Each of $f_{a, b}$ in the family is monotonic semiconjugate to the doubling map $D$ : $x \mapsto 2 x \bmod 1$ via a unique mapping $\varphi_{a, b}$ (see Lemma 3.8). When we say a continuous mapping of the circle is monotonic, we mean that its lift is a monotonic mapping of the real line. This mapping $\phi_{a, b}$ sends the periodic points of $f_{a, b}$ to the periodic points of the doubling map $D$ with the same period. Moreover, $(a, b) \mapsto \phi_{a, b}$ is continuous (see [4]).

In the following picture, graphs $(x, a(x))$ of the parameters $(a, 1)$ for which $x$ is a periodic point of $f_{a, 1}$ (with period $\left.\leq 10\right)$ are drawn in black.

Received by the editors July 4, 2009 and, in revised form, September 17, 2009; September 29, 2009; December 22, 2009; December 23, 2009 and December 26, 2009.

2010 Mathematics Subject Classification. Primary 37E10, 37F45; Secondary 37F30, 37C15, $37 \mathrm{C} 05$.

(C)2010 American Mathematical Society

Reverts to public domain 28 years from publication 


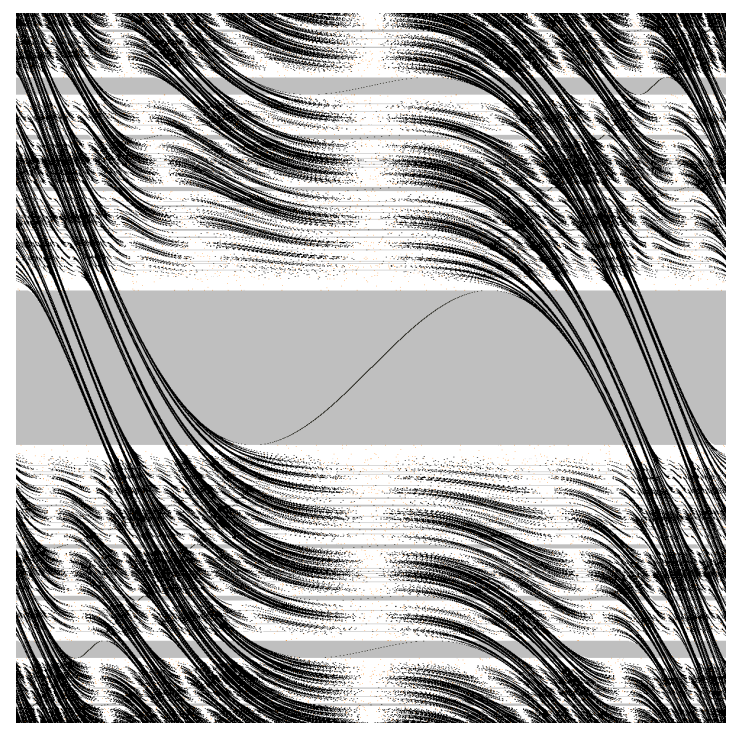

Indeed, all the functions $a(x)$ are defined in $\mathbb{T}^{1}$. The part of the graphs where the function decreases corresponds to a repulsive cycle, whereas increasing parts correspond to an attracting cycle. The intervals of values of $a$ for which $f_{a, 1}$ has an attracting cycle with period less than 10 are emphasized in grey.

One of the important facts concerning the mappings $f_{a, b}$ is that they correspond to mappings $g_{a, b}: \mathbb{S}^{1} \rightarrow \mathbb{S}^{1}$ of the complex circle (after conjugating by the exponential map) that extend to holomorphic functions $g_{a, b}: \mathbb{C}^{*} \rightarrow \mathbb{C}^{*}$ given by

$$
g_{a, b}(z)=e^{2 \sqrt{-1} \pi a} z^{2} e^{-b\left(z-\frac{1}{z}\right)} .
$$

These functions $g_{a, b}$ are symmetric with respect to the circle $\mathbb{S}^{1}=e^{2 \pi \sqrt{-1} \mathbb{T}^{1}} \subset \mathbb{C}^{*}$ in the sense that $g_{a, b}(1 / \bar{z})=1 / \overline{g_{a, b}(z)}$. Any $g_{a, b}$ has at most one attracting cycle on the circle, and if there is one, then all the critical points belong to the same component of the immediate basin. Since this component contains only one point of the cycle, it yields a naturally distinguished point of the cycle.

Definition 1.1. A tuple of parameters $(a, b) \in \mathbb{T}^{1} \times[0,1]$ is of type $\tau$ if $g_{a, b}$ has an attracting cycle on the circle with $\phi_{a, b}\left(x_{0}\right)=\tau$, where $x_{0}$ is the distinguished point of the attracting cycle of $g_{a, b}$.

Definition 1.2. The tongue $T_{\tau}$ of type $\tau \in \mathbb{T}^{1}$ is the set of parameters $(a, b) \in$ $\mathbb{T}^{1} \times[0,1]$ of type $\tau$.

Remark 1.3. Since any type $\tau$ is a periodic point of $D$, it is a quotient $\frac{k}{2^{p}-1}$ where $k \in\left\{0, \ldots, 2^{p}-2\right\}$ and $p$ is the period of the cycle.

The tongues $T_{\tau}$ are open disjoint subsets of $\mathbb{T}^{1} \times[0,1]$. Some of their properties were studied in $[5]$. 
The following picture shows the tongues (in black) in the parameter space $[-1 / 2,1 / 2] \times[0,1]:$

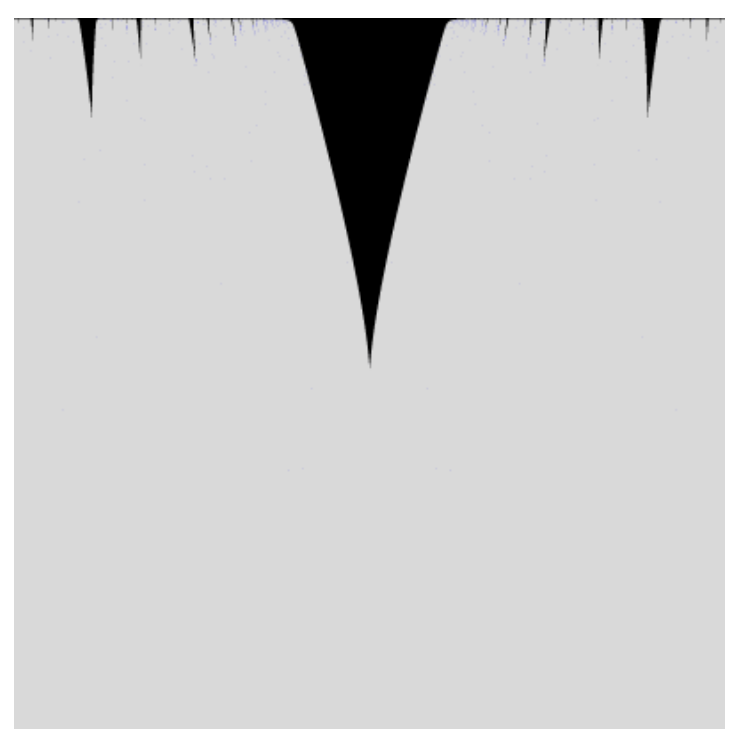

One of the questions left unanswered by the authors of 5 concerned the connectedness of the set $T_{\tau}$. We show here that the set $T_{\tau}$ is connected. We shall use quasiconformal techniques in the proof.

Theorem 1.4. For each type $\tau$ (periodic point of $D$ ) there exists a unique $\left(a_{\tau}, b_{\tau}\right) \in$ $T_{\tau}$ such that $f_{a_{\tau}, b_{\tau}}$ has a superattracting cycle. Then $b_{\tau}=1$, and all $(a, b) \in T_{\tau}$ can be joined to $\left(a_{\tau}, 1\right)$ by a continuous path in $T_{\tau}$.

Corollary 1.5. The tongues are connected.

The paths will be constructed by a quasiconformal deformation.

Section 3 is devoted to the construction of the deformation inside the tongue for parameters with no superattracting cycle; see Lemma 3.1. In section 4, it is shown that the deformation path tends to a unique limit parameter with a superattracting cycle.

\section{General properties of $g_{a, b}$}

We recall several properties concerning of the mappings $g_{a, b}$ that we will use later on.

We first study the set of critical points of these mappings. Let

$$
g(z)=z^{2} e^{u(1 / z)+v(z)}
$$

where $u$ and $v$ are polynomials of respective degrees $p \geq 1$ and $q \geq 1$. Since $\operatorname{deg} v \geq 1$, the set of essential singularities of $g$ contains 0 . The point $\infty$ is the only other essential singularity of $g$.

The following fact is used in Proposition 3.6.

Lemma 2.1. The derivative of $g$ is $g^{\prime}(z)=\frac{1}{z^{p-1}} P(z) e^{u(1 / z)+v(z)}$, where $P(z)=$ $z^{p+1} v^{\prime}(z)+2 z^{p}-z^{p-1} u^{\prime}(1 / z)$. The function $P(z)$ is a polynomial, its degree is $p+q$ and its constant term is equal to the leading term of $u$, which is not zero. In particular the critical points of $g$ are the roots of $P(z)$ with the same multiplicities. 
Now if $p=q=1$, let $(a, b, c) \in \mathbb{C} / \mathbb{Z} \times\left(\mathbb{C}^{*}\right)^{2}$ be defined by $v(z)+u(1 / z)=$ $2 \pi A \sqrt{-1}-(b z-c / z)$, with $a=A \bmod 1$. Then the critical points of $g$ are the roots of the quadratic polynomial $b z^{2}-2 z+c$, namely $\frac{1 \pm \sqrt{1-b c}}{b}$, where $\sqrt{1-b c}$ stands for an arbitrary choice of one of the two square roots of $1-b c$.

We will say that a mapping $f: \mathbb{C}^{*} \rightarrow \mathbb{C}^{*}$ is symmetric with respect to the circle if for all $z \in \mathbb{C}^{*}, f\left(\frac{1}{\bar{z}}\right)=\frac{1}{f(z)}$. If we suppose that $g$ has the above symmetry property, then $c=\bar{b}$ and $a \in \mathbb{R}$.

We now suppose that $p=q=1$ and that $g$ is symmetric with respect to the circle, and we denote it by $g_{a, b}$, i.e. $g_{a, b}(z)=\lambda z^{2} e^{-(b z-\bar{b} / z)}$, with $|\lambda|=1$. One must notice that there exists a unique rotation that conjugates $g_{a, b}$ to a function $g_{\tilde{a}, \tilde{b}}=$ $\tilde{\lambda} z^{2} e^{-(\tilde{b} z-\tilde{b} / z)}$ with $\tilde{b} \in \mathbb{R}_{+}$. Indeed, the only way one can do this is by conjugating by the linear map $z \mapsto \frac{|b|}{b} z$. This yields $\left(\frac{b}{|b|}\right)^{-1} g_{a, b}\left(\frac{|b|}{b} z\right)=\frac{|b| \lambda}{b} z^{2} e^{-(\tilde{b} z-\tilde{b} / z)}$ with $\tilde{b}=\frac{|b|}{b} \in \mathbb{R}_{+}$.

Lemma 2.2. Let $a \in \mathbb{T}^{1}$ and $b \in \mathbb{C}$. Then the mapping

$$
g_{a, b}(z)=e^{2 \pi a \sqrt{-1}} z^{2} e^{-(b z-\bar{b} / z)}
$$

has a monotonic restriction to $\mathbb{S}^{1}$ if and only if $|b| \leq 1$. In that case $|b|=1$ is equivalent to the fact that the critical points belong to the unit circle.

Proof. When $|b|<1$, the critical points of $g_{a, b}$, which are $\left\{\frac{1-\sqrt{1-|b|^{2}}}{b}, \frac{1+\sqrt{1-|b|^{2}}}{b}\right\}$, don't belong to the circle. From what follows we see that $g_{a, b \mid \mathbb{S}^{1}}$ is a monotonic mapping of the circle. From the continuous dependence of $g_{a, b}$ on $b$, it follows that for any $a$ the mapping $g_{a, b \mid \mathbb{S}^{1}}$ is also monotonic when $|b|=1$. In the latter case, the map has only one critical point $1 / b$, and this critical point belongs to the circle.

Conversely, suppose $|b|>1$. Here it is convenient to make the computations with the lifted map $f_{a,|b|}$ of the mapping $g_{a,|b|}$, which is conjugated with $g_{a, b}$ by a rotation. The critical points of $f_{a,|b|}$ are the solutions of $\cos (2 \pi x)=\frac{1}{|b|}$. These critical points all belong to the real axis, and the second derivative $f_{a,|b|}^{\prime \prime}(x)=-4 \pi|b| \sin (2 \pi x)$ does not vanish at these points. Consequently $f_{a,|b|}$, and hence $g_{a, b \mid \mathbb{S}^{1}}$, is not monotonic when $|b|>1$.

We now compute the asymptotic values of the mapping $g_{a, b}$. Recall that $\alpha$ is an asymptotic value of $g_{a, b}$ if there exists a continuous path $\eta:\left[0,+\infty\left[\rightarrow \mathbb{C}^{*}\right.\right.$ such that the limit of $\eta(t)$ when $t \rightarrow+\infty$ is either 0 or $\infty$ and such that $\alpha=\lim _{t \rightarrow+\infty} g_{a, b}(\eta(t))$.

Proposition 2.3. Let $\lambda \in \mathbb{S}^{1}, b \in \mathbb{R}^{*}$. Then the set of asymptotic values of $g(z)=\lambda z^{2} e^{-b(z-1 / z)}$ is $\{0, \infty\}$.

Proof. Consideration of the paths $t \mapsto \eta_{ \pm}(t)= \pm t$ shows that 0 and $\infty$ are asymptotic values. Following [8], we show that any asymptotic value $\alpha$ must be 0 or $\infty$.

By definition we have $\alpha=\lim _{t \rightarrow+\infty} g \circ \eta(t)$, for some path $\eta$ tending to 0 or $\infty$; and by symmetry, we may assume without loss of generality that $\eta(t) \rightarrow \infty$ as $t \rightarrow+\infty$.

Let $x, y:[0,+\infty[\rightarrow \mathbb{R}$ be defined by $x+\sqrt{-1} y=-b(\eta-1 / \eta)$.

If there exist $c \in \mathbb{R}$ and a sequence $t_{n} \rightarrow+\infty$ such that $x\left(t_{n}\right) \geq c$ for all $n$, then $g\left(\eta\left(t_{n}\right)\right) \rightarrow \infty$. So $\alpha \neq \infty$ implies that $x(t) \rightarrow-\infty$ as $t \rightarrow+\infty$. Let $\arg _{0}$ denote the 
determination of the argument that takes values into $[0,2 \pi]$. As a consequence of the above, we have $\arg _{0} \eta=\arg _{0}\left(\frac{x+\sqrt{-1} y}{-b}\right)+o(1) \in\left[\frac{\pi}{2}, \frac{3 \pi}{2}\right]$ for all $t$ large enough.

On the other hand, if there exist $t_{n} \rightarrow+\infty$ such that $\left(\left|y\left(t_{n}\right)\right|\right)_{n}$ is bounded, then $g\left(\eta\left(t_{n}\right)\right) \rightarrow 0$ as $n \rightarrow \infty$. Indeed, $\log |g|=\log |\eta|^{2}+x=\log \left(\frac{x^{2}}{b^{2}}+\frac{y^{2}}{b^{2}}\right)+x+o(1) ;$ thus $\log \left|g\left(\eta\left(t_{n}\right)\right)\right|=x_{n}+o\left(\left|x_{n}\right|\right)$. Since $x\left(t_{n}\right) \rightarrow-\infty$, we would have $g\left(\eta\left(t_{n}\right)\right) \rightarrow 0$ as $n \rightarrow \infty$. Consequently $\alpha \neq 0$ implies that $y$ is unbounded.

Let arg denote the imaginary part of the analytic continuation of one choice of $\log g$ along the path $\eta$. From the convergence of $\log g \circ \eta(t)$ as $t \rightarrow \infty$, it follows that $\arg g \circ \eta$ is bounded. Since $\arg g \circ \eta=y+\arg \lambda+2 \arg \eta, \arg \eta$ is unbounded. This contradicts the above.

One could also use the Ahlfors-Carleman-Denjoy theorem to show that $g_{a, b}$ has no asymptotic value in $\mathbb{C}^{*}$. The result follows from the fact that the entire function $f_{a, b}(z)=2 z-a+\frac{b}{\pi} \sin 2 \pi z$ has no finite asymptotic values. Note that we would use the fact that $f_{a, b}$ has finite growth order. Since we will need the definition of the growth order for the characterization of the double standard family, we recall it below.

Definition 2.4. Let $a$ be a point of the Riemann sphere $\mathbb{P}^{1}, U$ a neighbourhood of $a$ and $f: U \backslash\{a\} \rightarrow \mathbb{C}$ a holomorphic function defined in the punctured neighbourhood $U \backslash\{a\}$ of $a$ such that it has an essential singularity at $a$. Then the growth order of $f$ near $a$ is defined by

$$
\limsup _{r \rightarrow 0}-\frac{\log \log M_{a}(f, r)}{\log r}
$$

where $M_{a}(f, r)=\sup _{d(z, a)=r}|f(z)|$ and $d$ is the spherical distance.

This definition is independent of a change of chart in the domain. For the family of mappings $\left(g_{a, b}\right)_{a \in \mathbb{T}^{1}, b \in \mathbb{R}^{*}}$, the growth orders near 0 and near $\infty$ are both 1 .

Now we return to the problem of finding attracting basins for $g_{a, b}$.

Proposition 2.5. For any $(a, b) \in \mathbb{T}^{1} \times[0,1]$, the mapping $g_{a, b}$ can have at most two attracting cycles, and if there are, both are of the same period. Moreover, the immediate basin of an attracing cycle of $g_{a, b}$ contains at least one critical point.

If an attracting cycle of $g_{a, b}$ belongs to the circle, it attracts both critical points and there is no other attracting cycle. In that case, the critical points belong to the same component of the immediate basin.

Proof. Since the asymptotic values of $g_{a, b}$ don't belong to its set of definitions, the iterates $g_{a, b}^{\circ n}$ have the same asymptotic values as $g_{a, b}$.

Let $n \in \mathbb{N}^{*}$ be the period of the attracting cycle of $g_{a, b}$. Let $R>0$ be the maximal radius of the disks centered at 0 on which the inverse $\psi$ of the linearizing map corresponding to this attracting cycle can be defined. Let $r<R$ be such that $\frac{r}{|\lambda|}>R$.

If we suppose that $B_{r}:=\psi(D(0, r))$ does not contain any asymptotic value or critical value of $g_{a, b}^{\circ n}$, then $g_{a, b}^{\circ n}:\left(g_{a, b}^{\circ n}\right)^{-1}\left(B_{r}\right) \rightarrow B_{r}$ is a covering map. Since $B_{r}$ is simply connected it would produce an inverse branch of $g_{a, b}^{\circ n}$ defined on the 
whole set $B_{r}$. Then $\psi$ could be extended in $D\left(0, \frac{r}{|\lambda|}\right)$ by the formula $\psi(z)=$ $\left(g_{a, b}^{\circ n}\right)^{-1}(\psi(\lambda z))$, which contradicts the maximality of $R<\frac{r}{|\lambda|}$.

We conclude that $B_{\varepsilon}$ contains a critical or an asymptotic value of $g_{a, b}^{\circ n}$. But it cannot be an asymptotic value because the asymptotic values of $g_{a, b}^{\circ n}$ do not belong to the domain of definition of the map. Hence, the basin of the attracting cycle contains at least one critical point.

Since there are only two critical points, there are at most two attracting cycles. Symmetry implies they both have the same period. Moreover, if this cycle belongs to the circle, from the symmetry property of $g_{a, b}$ it follows that both critical points belong to its immediate basin.

\section{Deformation}

Lemma 3.1. Let $(a, b) \in \mathbb{T}^{1} \times[0,1]$ be such that $g_{a, b}$ has an attracting cycle on the circle of type $\tau$ with multiplier $\lambda$. Then there exists a family $\left(\varphi_{\rho}\right)_{\rho \in] 0,1[}$ of quasiconformal homeomorphisms $\varphi_{\rho}: \mathbb{P}^{1} \rightarrow \mathbb{P}^{1}$ of $\mathbb{P}^{1}=\mathbf{P}^{\mathbf{1}}(\mathbb{C})$ fixing 0 and $\infty$, depending analytically on the real variable $\rho$, such that $\varphi_{\lambda}=i d_{\mathbb{P}^{1}}$ and such that $\varphi_{\rho}$ conjugates $g_{a, b}$ to some $g_{a_{\rho}, b_{\rho}}$ which has an attracting cycle on the circle of type $\tau$ with multiplier $\rho$ :

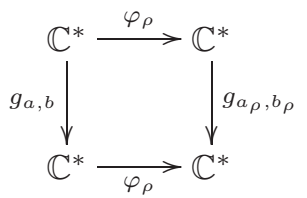

Let us show Lemma 3.1 The aim is to construct a mapping $g_{\tilde{a}, \tilde{b}}$, belonging to the complex double standard family, from $g:=g_{a, b}$, such that $g_{\tilde{a}, \tilde{b}}$ has the same type as $g:=g_{a, b}$ but with a given multiplier $\left.\rho \in\right] 0,1[$. This is done by quasiconformal deformation.

3.1. Deformation process. Let $\varphi$ be a local linearizing map of $g^{\circ p}$, where $p$ is the period of the attracting cycle, defined in a neighbourhood of $x \in \mathbb{S}^{1}$, where $x$ is the point of the cycle which lies in the same Fatou component as the critical points of $g$.

We will suppose that the derivative of $\varphi$ at $x$ satisfies $\left|\varphi^{\prime}(x)\right|=1$ and that it is tangent to the unit circle $\mathbb{S}^{1}$ and points towards the anticlockwise direction. The mapping $\varphi$ is uniquely determined by this normalization. We have $\varphi \circ g^{\circ p}=\lambda \varphi$, $\varphi(x)=0$ and $\varphi^{\prime}(x)=\sqrt{-1} x$.

It is easy to see that $\varphi$ is symmetric (cf. Lemma 3.2 below), from which it follows that if $D_{R}$ is a disk centered in $0=\varphi(x)$ on which $\varphi^{-1}$ is well-defined, then $U=\varphi^{-1}\left(D_{R}\right)$ is stable by $z \mapsto 1 / \bar{z}$.

Lemma 3.2. Suppose $f$ is a holomorphic function defined in a neighbourhood of the circle, which is symmetric with respect to the circle, i.e. such that $f(1 / \bar{z})=1 / \overline{f(z)}$, and that it has an attracting cycle $\left(x, f(x), \ldots, f^{\circ p-1}(x)\right)$ in $\mathbb{S}^{1}$ with multiplier $\lambda$.

Then $\lambda \in \mathbb{R}$ and the linearizing mapping $\varphi$ defined in a neighbourhood of $x$ and with $\varphi^{\prime}(x)=\sqrt{-1} x$ satisfies $\overline{\varphi(1 / \bar{z})}=\varphi(z)$, and the preimages by $\varphi$ of any disk centered in 0 are symmetric with respect to the circle. 
Proof. Since $f$ is symmetric with respect to $\mathbb{S}^{1}$, the multiplier $\lambda$ is real. If we set $\psi(z)=\overline{\varphi(1 / \bar{z})}$, then it's easy to see that $\psi \circ f=\lambda \psi$ because $\lambda$ is real. Moreover, $\psi$ is holomorphic, and a simple computation shows that $\psi^{\prime}(x)=-\left(\frac{1}{x}\right)^{2} \overline{\varphi^{\prime}(1 / \bar{x})}=$ $\varphi^{\prime}(x)$.

Therefore, from the uniqueness of $\varphi$, it follows that $\varphi(1 / \bar{z})=\overline{\varphi(z)}$.

Then $\varphi(z) \in D_{R} \Leftrightarrow \overline{\varphi(z)} \in D_{R} \Leftrightarrow \varphi(1 / \bar{z}) \in D_{R}$.

We first use a quasiconformal deformation $\chi$ which transforms $D_{R}$ into $D_{r}$, with $R$ chosen so as to produce a deformed map with the expected multiplier $\rho$.

Lemma 3.3. Let $\alpha>0$ and

$$
\begin{aligned}
\chi: \mathbb{C}^{*} & \longrightarrow \mathbb{C}^{*}, \\
z & \longmapsto|z|^{\alpha} z .
\end{aligned}
$$

Then

- $\chi$ is a real-analytic diffeomorphism with $\chi\left(r e^{i \theta}\right)=r^{\alpha+1} e^{i \theta}$, and $\chi\left(z z^{\prime}\right)=$ $\chi(z) \chi\left(z^{\prime}\right)$

- we have

$$
\mu_{\chi}:=\frac{\partial \chi / \partial \bar{z}}{\partial \chi / \partial z}=\frac{\alpha / 2}{1+\alpha / 2} \frac{z}{\bar{z}}
$$

In particular $\left|\mu_{\chi}\right|$ is constant and $\left\|\mu_{\chi}\right\|_{\infty}=\left|\frac{\alpha / 2}{1+\alpha / 2}\right|$;

- if $\alpha=\frac{\log r}{\log R}-1, \chi$ sends the disk $D_{R}$ (of radius $R$ ) onto the disk $D_{r}$;

- if, moreover, $R, r<1$, then

$$
\left|\mu_{\chi}\right|=\frac{|1-\log r / \log R|}{1+\log r / \log R}<1 .
$$

We have the commutative diagram

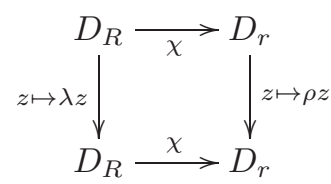

with $\rho=\chi(\lambda)=\lambda^{1+\alpha} \quad$ (in particular $\left.0<\rho<1\right)$.

Proof.

- The mapping $\chi$ is monotonic on the radii, $\chi(0)=0$ and $\chi(R)=R^{\frac{\log r}{\log R}}=r$.

- We have $\frac{\partial|z|}{\partial z}=\frac{1}{2} \frac{\bar{z}}{|z|}$ and $\frac{\partial|z|}{\partial \bar{z}}=\frac{1}{2} \frac{z}{|z|}$. Hence $\frac{\partial \chi}{\partial \bar{z}}=\frac{\alpha}{2}|z|^{\alpha-2} z^{2}$ and $\frac{\partial \chi}{\partial z}=$ $\left(\frac{\alpha}{2}+1\right)|z|^{\alpha}$.

- From the first point, we have $\chi(\lambda z)=\chi(\lambda) \chi(z)=\rho \chi(z)$.

Lemma 3.4. The composition

$$
U \stackrel{\varphi}{\longrightarrow} D_{R} \stackrel{\chi}{\longrightarrow} D_{r}
$$


induces a differential Beltrami form $\sigma_{\rho}$ on $U$, depending analytically on $\rho$, which is invariant by $g$ :

$$
\begin{aligned}
\sigma_{\rho} & =\mu_{\rho} \frac{d \bar{z}}{d z} \\
& =\frac{\alpha / 2}{1+\alpha / 2} \cdot \frac{\varphi(z)}{\overline{\varphi(z)}} \cdot \frac{\overline{\varphi^{\prime}(z)}}{\varphi^{\prime}(z)} \cdot \frac{d \bar{z}}{d z}
\end{aligned}
$$

satisfying $\mu_{\rho}(1 / \bar{z})=\overline{\mu_{\rho}(z)}$ on $U$.

Proof. The complex dilatation $\mu_{\rho}$ is the coefficient of the Beltrami form $\frac{\partial \chi \circ \varphi}{\partial \chi \circ \varphi}$. Analytic dependence follows from (3) and the fact that $\alpha=\frac{\log \rho}{\log \lambda}-1$.

Invariance by $g$ follows from (3) and (5) above.

Thus, we have a differential Beltrami form $\sigma_{\rho}$ on a symmetric open set (image of a disk $D_{R}$ under $\varphi^{-1}$ ), which is invariant by $g$ with the symmetric complex dilatation coefficient $\mu_{\rho}$ (i.e. $\left.\mu_{\rho}(1 / \bar{z})=\overline{\mu_{\rho}}(z)\right)$.

We propagate $\sigma_{\rho}$ over almost all the attracting basin via pullback by $g$. The resulting Beltrami form is still symmetric, and we can extend it by 0 outside the basin.

To do so, the Beltrami form $\sigma_{\rho}=\mu_{\rho} \frac{d \bar{z}}{d z} \in L^{\infty}$ is constructed in this manner (compare [7]) :

- on $U, \sigma_{\rho}$ is the $g$-invariant differential Beltrami form given by Lemma 3.4 it is invariant by $g$;

- on the preimages $g^{-n}(U)$ of $U$, the form $\sigma_{\rho}$ is given by

$$
\sigma_{\rho}(z)=\frac{\overline{g^{\circ n^{\prime}}(z)}}{g^{\circ n^{\prime}}(z)} \mu_{\rho}\left(g^{\circ n}(z)\right) \frac{d \bar{z}}{d z}
$$

where $n$ is such that $g^{\circ n}(z) \in U$. Note that this is well defined because $\sigma_{\rho}$ is $g$-invariant on $U$;

- elsewhere, $\sigma_{\rho}$ vanishes.

From holomorphy of $g$ it follows that $\left\|\sigma_{\rho}\right\|_{\infty}=\left\|\sigma_{\rho}\right\|_{L^{\infty}\left(\mathbb{P}^{1}\right)}=\left\|\sigma_{\rho}\right\|_{L^{\infty}(U)}$.

The coefficient $\mu_{\rho}$ (or $\sigma_{\rho}$ ) is easily seen to be symmetric on $U$. Moreover, $\forall n$, $\forall z, g^{\circ{ }^{\prime \prime}}(1 / \bar{z})=\overline{g^{\circ{ }^{\prime}}(z)}$. It follows that for $z$ such that $g^{\circ n}(z) \in U$,

$$
\mu_{\rho}(1 / \bar{z})=\frac{\overline{g^{\circ{ }^{\prime \prime}}(1 / \bar{z})}}{g^{\circ n^{\prime}}(1 / \bar{z})} \mu_{\rho}\left(g^{\circ n}(1 / \bar{z})\right)=\overline{\left(\frac{\overline{g^{\circ{ }^{\prime \prime}}(z)}}{g^{\circ n^{\prime}}(z)} \mu_{\rho}\left(g^{\circ n}(z)\right)\right)}=\overline{\mu_{\rho}(z)} .
$$

Remark 3.5. The above pullback discussion is uniform in $\rho$. As a consequence, the Beltrami form $\sigma_{\rho}$ depends analytically on $\rho$.

Since $\left\|\sigma_{\chi}\right\|_{\infty}=\left\|\sigma_{\rho}\right\|_{\infty}<1$, the Riemann measureable mapping theorem yields the existence of a unique quasiconformal homeomorphism $\Phi_{\rho}:\left(\mathbb{P}^{1}, 0, \infty, x\right) \longrightarrow$ $\left(\mathbb{P}^{1}, 0, \infty, x\right)$ such that the Beltrami form $\sigma_{\rho}$ is $\Phi_{\rho}$-invariant. Moreover, this homeomorphism is symmetric with respect to the circle (compare section 2), and by the analytic dependence on parameters (compare for example [1]), $\rho \mapsto \Phi_{\rho}(z)$ is real-analytic for any $z \in \mathbb{P}^{1}$. 
From the $g$-invariance of $\sigma_{\rho}$, it follows that $\tilde{g}:=\Phi_{\rho} \circ g \circ \Phi_{\rho}^{-1}$ and that $\tilde{\varphi}:=$ $\left(\Phi_{\rho} \circ \varphi^{-1} \circ \chi\right)^{-1}$ are holomorphic on their respective domains of definition:

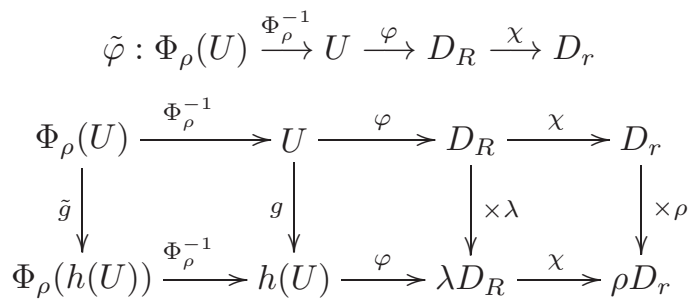

The new mapping $\tilde{g}$ has an attracting cycle of period $p$, like $g$, with a local linearizing mapping $\tilde{\varphi}$ defined in the neighbourhood of the point $\Phi_{\rho}(x)$ (which belongs to this cycle). The new multiplier is thus $\rho=\lambda^{1+\alpha}$. Note that all values in the interval $] 0,1[$ can be assigned to $\rho$.

We need to see that we obtained a mapping which belongs to the family of complexified double standard maps $\left(g_{a, b}\right)_{a, b}$. In the next section, we will see that, after conjugating by a suitable rotation, the new map belongs to the family.

3.2. Back to the double standard family. We now check that the mapping $\tilde{g}$ constructed in the previous section is conjugated by a rotation $R$ to a unique element $g_{\tilde{a}, \tilde{b}}$ in the family of mappings $\left(g_{a, b}\right)_{a, b}$. This is a consequence of the following and of the discussion in section 2 about the existence and unicity of the conjugacy by a rotation.

Proposition 3.6. Let $(a, b, c) \in \mathbb{C} / \mathbb{Z} \times \mathbb{C}^{*} \times \mathbb{C}^{*}$ and let $g_{a, b, c}: \mathbb{C}^{*} \rightarrow \mathbb{C}^{*}$ be defined by

$$
g_{a, b, c}(z)=e^{2 \sqrt{-1} \pi a} z^{2} e^{-(b z-c / z)} .
$$

Let $\varphi, \psi$ be orientation-preserving homeomorphisms of the Riemann sphere $\mathbb{P}^{1}$ fixing 0 and $\infty$.

If $\psi \circ g_{a, b, c} \circ \varphi: \mathbb{C}^{*} \rightarrow \mathbb{C}^{*}$ is holomorphic, then there exist $(\alpha, \beta, \gamma) \in \mathbb{C} / \mathbb{Z} \times \mathbb{C}^{*} \times \mathbb{C}^{*}$ such that $\psi \circ g_{a, b, c} \circ \varphi=g_{\alpha, \beta, \gamma}$.

If we suppose that $g_{a, b, c}$ is a symmetric map with respect to the circle, i.e. $g _ { a , b , c } ( 1 / \overline { z } ) = 1 \longdiv { g _ { a , b , c } ( z ) }$, hence that $a \in \mathbb{T}^{1}$ and $c=\bar{b}$ and that $\varphi$ and $\psi$ are also symmetric with respect to the circle, then the mapping $g_{\alpha, \beta, \gamma}$ is also symmetric, $\alpha \in \mathbb{T}^{1}, \gamma=\bar{\beta}$, and we have $|\beta|<1$ if and only if $|b|<1$ and $|\beta|=1$ if and only if $|b|=1$.

Proof. Without loss of generality we can suppose $\varphi$ and $\psi$ are quasiconformal. Indeed, let $V$ be the set of critical values of $g_{a, b, c}$. It contains one or two points, and $\psi(V)$ is the set of critical values of $h:=\psi \circ g_{a, b, c} \circ \varphi$. From the finiteness of $V$ and the compactness of $\mathbb{P}^{1}$, it follows that there exists an isotopy $\psi_{t}: \mathbb{P}^{1} \rightarrow \mathbb{P}^{1}$ between $\psi=\psi_{0}$ and a quasiconformal homeomorphism $\psi_{1}$ such that, for all $t \in[0,1]$, $\psi_{t}(0)=0, \psi_{t}(\infty)=\infty$ and such that for all $v \in V, \psi_{t}(v)=\psi(v)$. Thanks to this last property, the isotopy $\psi_{t}$ can be lifted up into an isotopy $\varphi_{t}$ starting from $\varphi_{0}=\varphi$. The final function $\varphi_{1}$ of this isotopy is quasiconformal since it is locally a composition of conformal or quasiconformal mappings.

Since $\varphi$ and $\psi$ are orientation-preserving homeomorphisms of $\mathbb{C}^{*}$, they induce the identity map on $H_{1}\left(\mathbb{C}^{*}, \mathbb{Z}\right)$, the first integral homology group of $\mathbb{C}^{*}$. Since 
$H_{1}\left(\mathbb{C}^{*}, \mathbb{Z}\right)$ is generated by the homology class $[\gamma]$ of a curve $\gamma$ winding one time round 0 in the anticlockwise direction, we have

$$
\frac{1}{2 \sqrt{-1} \pi} \int_{(0,+)} \frac{h^{\prime}(z)}{h(z)} d z=\frac{1}{2 \sqrt{-1} \pi} \int_{(0,+)} \frac{g_{a, b, c}^{\prime}(z)}{g_{a, b, c}(z)} d z .
$$

The latter depends continuously on $(a, b, c) \in \mathbb{C} / \mathbb{Z} \times \mathbb{C} \times \mathbb{C}$. Consequently, it is constant when $(a, b, c)$ varies and we have

$$
\frac{1}{2 \sqrt{-1} \pi} \int_{(0,+)} \frac{h^{\prime}(z)}{h(z)} d z=\frac{1}{2 \sqrt{-1} \pi} \int_{(0,+)} \frac{g_{0,0,0}^{\prime}(z)}{g_{0,0,0}(z)} d z=2 .
$$

It follows that there exists a holomorphic function $u: \mathbb{C}^{*} \rightarrow \mathbb{C}^{*}$ such that $h(z)=$ $z^{2} e^{u(z)}$. In order to characterize the function $u$, we need to know the growth order of $h$ near 0 and $\infty$.

From now on, we suppose that $\varphi$ and $\psi$ are $K$-quasiconformal homeomorphisms of $\mathbb{P}^{1}$. It is well known that such mappings are locally Hölder continuous of exponent $1 / K$. So, by applying this to the inverse mapping of $\varphi$ near $\infty$, we can find $R_{\infty}>0$ and $C_{\infty}>0$ such that for all $z$ satisfying $|\varphi(z)| \geq R_{\infty}$, we have $|\varphi(z)| \leq C_{\infty}|z|^{K}$. Moreover, we can fix $R_{\infty}$ and $C_{\infty}$ such that the same holds if we only change $\varphi$ and replace it by $\psi$.

We concentrate on estimating the growth order of $h$ near $\infty$. Since $\varphi(z) \rightarrow \infty$ when $z \rightarrow \infty$, we can suppose that $|z|$ is such that $|\varphi(z)| \geq R_{\infty}$. Then

$$
\begin{aligned}
\operatorname{Re}\left(-\left(b \varphi(z)+\frac{c}{\varphi(z)}\right)\right) & \leq|b||\varphi(z)|+\frac{|c|}{|\varphi(z)|} \\
& \leq|b| C_{\infty}|z|^{K}+\frac{|c|}{R_{\infty}} .
\end{aligned}
$$

Hence $|g \circ \varphi(z)| \leq C|z|^{2 K} e^{C^{\prime}|z|^{K}}$, for some positive constants $C, C^{\prime}$. Therefore if $\left|\psi \circ g_{a, b, c} \circ \varphi(z)\right| \geq R_{\infty}$, we have $|h(z)|=\left|\psi \circ g_{a, b, c} \circ \varphi(z)\right| \leq C^{\prime \prime}|z|^{2 K^{2}} e^{C^{\prime \prime \prime}|z|^{K}}$ for some other positive constants $C^{\prime \prime}, C^{\prime \prime \prime}$. Thus the growth order of $h$ near $\infty$ is finite.

This conclusion also holds near 0 from a similar argument using the Hölder continuity of $\varphi$ near 0 .

Therefore $u$ has a non-zero Laurent series development,

$$
u(z)=\sum_{n=-p}^{q} a_{n} z^{n}
$$

with $(p, q) \in \mathbb{N}^{*} \times \mathbb{N}^{*}, a_{-p} \neq 0$ and $a_{q} \neq 0$.

From the definition of $h$, it follows that $h$ has the same number of critical points as $g_{a, b, c}$. From section 2 we know that the mapping $g_{a, b, c}$ has two critical points counting multiplicity.

We have

$$
\begin{aligned}
h^{\prime}(z) & =\left(2 z+z^{2} u^{\prime}(z)\right) e^{u(z)} \\
& =\frac{1}{z^{p-1}}\left(2 z^{p}+z^{p+1} u^{\prime}(z)\right) e^{u(z)} .
\end{aligned}
$$

The function $P(z)=\left(2 z^{p}+z^{p+1} u^{\prime}(z)\right)$ is a polynomial of exact degree $p+q$ such that $P(0) \neq 0$; thus $h$ has $p+q$ critical points counting multiplicity. Therefore we have $p=q=1$. This ends the proof of the first part of Proposition 3.6. 
The symmetry part of the last assertion is straightforward, while the equivalences on the bound $|\beta|<1$ or the identity $|\beta|=1$ follows from Lemma 2.2 .

From this proposition, it follows that there exist $(\alpha, \beta) \in \mathbb{T}^{1} \times \overline{\mathbb{D}}$ depending on $\rho$ such that $\Phi_{\rho} \circ g \circ \Phi_{\rho}^{-1}=e^{2 \sqrt{-1} \pi a} z^{2} e^{-(\beta z-\bar{\beta} / z)}$. Moreover, we have seen in section 2 that there exists a unique rotation $R$ such that $R^{-1} \circ \Phi_{\rho} \circ g \circ \Phi_{\rho}^{-1} \circ R$ belongs to the family of mappings $\left(g_{a, b}\right)_{a \in \mathbb{T}^{1}, b \in[0,1]}$.

3.3. Type of the deformed cycle. Given any $\rho \in] 0,1[$, we have a process for constructing a map in the double standard family by deformation of a map belonging to a tongue, such that the new map has an attracting cycle with multiplier $\rho$. We check that this construction does not change the type.

Proposition 3.7. The tuple of parameters $(\tilde{a}, \tilde{b})$ corresponding to the new function $\tilde{g}$ is of type $\tau$.

Proof. Let's recall that $\tau$ is the image by the map $\phi_{\tilde{a}, \tilde{b}}(x)=\lim _{n \rightarrow \infty} F_{a, b}^{\circ n}(x) / 2^{n}$ (see [4]) of the point $\tilde{x}$ of the attracting cycle of $g_{\tilde{a}, \tilde{b}}$ which belongs to the basin component that contains the critical points.

The proposition is a consequence of the following uniqueness property of the semiconjugacy $\phi_{a, b}$.

Lemma 3.8. Let $f: \mathbb{T}^{1} \rightarrow \mathbb{T}^{1}$ be a monotonic continuous mapping. If $\varphi, \psi: \mathbb{T}^{1} \rightarrow$ $\mathbb{T}^{1}$ are non-decreasing continuous mappings of degree 1 such that $\varphi \circ f=2 \times \varphi$ and $\psi \circ f=2 \times \psi$, then $\varphi=\psi$.

Remark 3.9. In the above lemma, the continuity assumption is not necessary, but, if one considers discontinuous mappings, the "non-decreasing" assumption has to be restated in order to take that specific context into account.

Proof. Let $F$ be a lift of $f$, and let $\tilde{\varphi}$ be a lift of $\varphi$. Since $\tilde{\varphi} \circ F$ is a non-decreasing real function, it is a lift of $2 \varphi$.

Therefore, there exists an integer constant $k$ such that

$$
\forall x \in \mathbb{R}, \tilde{\varphi}(F(x))=2 \tilde{\varphi}(x)+k .
$$

It follows that if we take $\varphi_{1}=\tilde{\varphi}+k$, then we have $\varphi_{1} \circ F=2 \varphi_{1}$. We assume that we have the same properties for a lift $\psi_{1}$ of $\psi$.

The mappings $\varphi_{1}$ and $\psi_{1}$ are both non-decreasing of degree 1 and locally bounded; thus, the function $\varphi_{1}-\psi_{1}$ is periodic and bounded. But then

$$
\varphi_{1}(x)-\psi_{1}(x)=\frac{1}{2}\left(\varphi_{1}(F(x))-\psi_{1}(F(x))\right),
$$

so

$$
\varphi_{1}(x)-\psi_{1}(x)=\left(\frac{1}{2}\right)^{n}\left(\varphi_{1}\left(F^{\circ n}(x)\right)-\psi_{1}\left(F^{\circ n}(x)\right)\right),
$$

for all $n$. Consequently, $\varphi_{1}(x)-\psi_{1}(x)=0$. 
The following diagram is commutative:

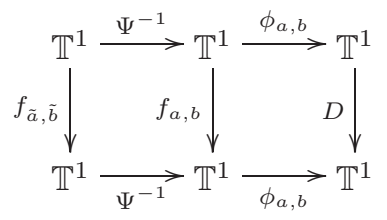

where $\Psi$ is defined by $\exp \circ \Psi=\Phi_{\mid \mathbb{S}^{1}} \circ$ exp. Therefore, we have $\phi_{\tilde{a}, \tilde{b}}=\phi_{a, b} \circ \Psi^{-1}$. As a consequence $(\tilde{a}, \tilde{b})$ is of type $\tau$.

\section{PATH}

Lemma 3.3 gives the value of the multiplier $\rho$ of the new mapping $g_{\tilde{a}, \tilde{b}}$; it is $\rho=\lambda^{1+\alpha}$, where $\alpha$ is taken in the interval $]-1,+\infty[$. It is then clear that all values in the interval ] $0,1[$ can be assigned to $\rho$ when we change the parameter of our construction (it suffices to set $\alpha=\frac{\log \rho}{\log \lambda}-1$ ).

Define $\gamma(\rho):=(\tilde{a}(\rho), \tilde{b}(\rho))$, where $(\tilde{a}(\rho), \tilde{b}(\rho))$ is the tuple of parameters corresponding to $f_{\tilde{a}, \tilde{b}}$, which is the restriction of $g_{\tilde{a}, \tilde{b}}$ to the unit circle (where $g_{\tilde{a}, \tilde{b}}$ was constructed from $g_{a, b}$ by the process of quasiconformal deformation described above).

This mapping $\gamma$ is well defined on $] 0,1[$, satisfies $\gamma(\lambda)=(a, b)$ and takes values into the type $\tau$ tongue $T_{\tau}$.

This mapping $\rho \mapsto \gamma(\rho)$ will be shown to be continuous. Indeed, it is realanalytic. Afterwards, it remains to show that $\gamma(0)$ can be defined and that it corresponds to a parameter with a superattracting cycle of type $\tau$.

4.1. Analyticity. The proof of the analytic dependence in $\rho$ of $(\tilde{a}, \tilde{b})=(a(\rho), b(\rho))$ is based on the study of the regularity of the dependence of the critical points and critical values of $\tilde{g}$, the resulting map of the quasiconformal deformation, and of the rotation which turns $\tilde{g}$ into a member of the family (cf. Proposition 3.6).

Recall that $\rho \mapsto \Phi_{\rho}(z)$ is a real-analytic map for all $z$. The critical points of $\tilde{g}_{\rho}=\Phi_{\rho} \circ g \circ \Phi_{\rho}^{-1}$, which are topologically characterized, are the images of the critical points of $g$ by $\Phi_{\rho}$. Let $\omega$ be one of the two critical points of $g$ and $\tilde{\omega}_{\rho}:=\Phi_{\rho}(\omega)$. Since $\rho \mapsto \Phi_{\rho}(\omega)$ is real-analytic, the critical point $\tilde{\omega}_{\rho}$ depends analytically on $\rho$.

The aim of the conjugacy by the rotation $R_{\rho}$ is to normalize $\tilde{g}_{\rho}$ so as to have a mapping $g_{\rho}=R_{\rho} \circ \tilde{g}_{\rho} \circ R_{\rho}^{-1}$ belonging to the complexified double standard family. Since the critical points of $\tilde{g}_{\rho}(z)=\lambda z^{2} e^{-(b z-\bar{b} / z)}$ are $\frac{1 \pm \sqrt{1-|b|^{2}}}{b} \in \frac{1}{b} \mathbb{R}$, it comes to move the critical point on the real positive half-line. Specifically, $R_{\rho} z=\frac{\left|\tilde{\omega}_{\rho}\right|}{\tilde{\omega}_{\rho}} z$.

Therefore, the corresponding critical point of $g_{\rho}: \omega_{\rho}=R_{\rho} \tilde{\omega}_{\rho}=\left|\tilde{\omega}_{\rho}\right|$ still depends analytically on $\rho$.

From the formulas of the critical points of $g_{\rho}=g_{a(\rho), b(\rho)}$, we get

$$
b(\rho)=\frac{2 \omega_{\rho}}{1+\omega_{\rho}^{2}},
$$

which is true for both of the critical points $\omega_{\rho}$.

We can express $a(\rho)$ in terms of $\rho$ by using the expression of the critical value of $g_{\rho}$, which is at the same time the image of $\omega_{\rho}$ by $g_{\rho}(z)=e^{2 \pi a(\rho) \sqrt{-1}} z^{2} e^{-b(\rho)(z-1 / z)}$ 
and the image of $h(\omega)$ by $R_{\rho} \circ \Phi_{\rho}$. We have

$$
e^{2 \pi a(\rho) \sqrt{-1}}=\frac{R_{\rho} \circ \Phi_{\rho}(f(\omega))}{\omega_{\rho}^{2} e^{-b(\rho)\left(\omega_{\rho}-1 / \omega_{\rho}\right)}} .
$$

So, $\rho \mapsto(a(\rho), b(\rho))$ is an analytic path from $] 0,1\left[\right.$ into $T_{\tau} \subset \mathbb{T}^{1} \times[0,1]$.

4.2. Ending of the path when the multiplier tends to 0. We have shown that, given a parameter $(a, b)=(a(0), b(0))$ in the type $\tau$ tongue, there exists a continuous path $\rho \in] 0,1[\mapsto \gamma(\rho)=(a(\rho), b(\rho))$ in that tongue, along one direction of which the multiplier of the type $\tau$ cycle is $\rho$ and tends to zero. We have to show that this path ends up at a definite limit when $\rho \rightarrow 0$, that this limit corresponds to a type $\tau$ parameter with superattracting cycle and that it doesn't depend on the starting point in the tongue.

We first show that the limit $\lim _{\rho \rightarrow 0}(a(\rho), b(\rho))$ exists.

Note that $b(\rho) \leq 1$ for all $\rho$ since for $b>1, f_{a, b}$ is not order-preserving and thus cannot be topologically conjugated to an order-preserving map.

Since $f_{a, b}^{\prime}(x) \geq 2(1-b)$ for all $(a, b, x)$ and $\lim _{\rho \rightarrow 0}\left(f_{a(\rho), b(\rho)}^{\circ p}\right)^{\prime}(x(\rho))=\lim _{\rho \rightarrow 0} \rho=0$, where $x(\rho)$ is any of the points of the attracting cycle (of period $p$ ) of $f_{a(\rho), b(\rho)}$, the value of $b(\rho)$ tends to 1 when $\rho \rightarrow 0$. In particular, from the continuous dependence of the semiconjugacy $\phi_{a, b}$ with respect to $(a, b)$, it follows that for any limit point of $(a(\rho), b(\rho))$ when $\rho \rightarrow 0$ the corresponding map has a superattracting cycle of exact period $p$.

The set $\Lambda$ of limit points of $\gamma(\rho)=(a(\rho), b(\rho))$ when $\rho \rightarrow 0$ is then included in the set of points $(a, 1) \in \mathbb{T}^{1} \times\{1\}$. This set $\Lambda$ is compact and connected since it is the decreasing intersection of the connected compact sets $\overline{\gamma(10,1 / n[)}$.

Thus $\Lambda$ is a point or contains an open set $] a_{1}, a_{2}\left[\right.$ in $\mathbb{T}^{1} \times\{1\}$. We will prove that its interior is empty.

To proceed we need the following lemma, also used later.

Lemma 4.1. For any $p \in \mathbb{N}$, the mapping $\mathbb{T}^{1} \ni a \mapsto f_{a, 1}^{\circ p}(1 / 2) \in \mathbb{T}^{1}$ is strictly increasing and of degree $2^{p}-1$.

Proof. Monotonicity comes from the fact that $\frac{\partial}{\partial a}\left(F_{a, 1}^{\circ p}(1 / 2)\right) \geq 1$, which can be seen by a direct calculation (compare [5]).

Now, it is sufficent to see that for all $(a, b, x) \in \mathbb{R} \times[0,1] \times \mathbb{R}$ and all $p \in \mathbb{N}$,

$$
F_{a+1, b}^{\circ p}(x)=F_{a, b}^{\circ p}(x)+2^{p}-1
$$

(recall that $f_{a, b}: \mathbb{T}^{1} \rightarrow \mathbb{T}^{1}$ is the quotient in $\mathbb{T}^{1}$ of the function $F_{a, b}: \mathbb{R} \rightarrow \mathbb{R}$ ).

This is true for $p=1$; moreover, an induction argument on $p$ gives

$$
\begin{aligned}
F_{a+1, b}^{\circ p+1}(x) & =F_{a+1, b}\left(F_{a+1, b}^{\circ p}(x)\right) \\
& =F_{a, b}\left(F_{a, b}^{\circ p}(x)+2^{p}-1\right)+1 \\
& =F_{a, b}^{\circ p+1}(x)+2^{p+1}-1 .
\end{aligned}
$$

Now we see that the limit is unique. Otherwise, $1 / 2$, which is the only critical point of $g_{a, 1}$, would be a $p$-periodic point for all of the maps $f_{a, 1}$ such that $a \in$ ]$a_{1}, a_{2}[$, which is impossible by the above. The limit is thus unique.

Secondly, the type of the limit $(a, 1)$ must be $\tau$. Indeed it has a type $\tau^{\prime}$. But since the set of parameters of a given type is an open set in $\mathbb{T}^{1} \times[0,1]$, all the values of the path close enough to $(a, 1)$ have the same type $\tau^{\prime}$, which implies that $\tau^{\prime}=\tau$. 
Lemma 4.2. Let $p \in \mathbb{N}^{*},(a, b) \in \mathbb{T}^{1}$ and $X \in \mathbb{R}$. Let $k \in \mathbb{N}$ such that $k<2^{p}-1$ and let $x=X \bmod 1 \in \mathbb{T}^{1}$. If $F_{a, b}^{\circ p}(X)=X+k$, then $\phi_{a, b}(x)=\frac{k}{2^{p}-1}$.

Proof. We have

$$
\begin{aligned}
\phi_{a, b}(x) & =\left(\lim _{n \rightarrow \infty} \frac{F_{a, b}^{\circ n}(X)}{2^{n}}\right) \bmod 1 \\
& =\left(\lim _{n \rightarrow \infty} \frac{F_{a, b}^{\circ n p}(X)}{2^{n p}}\right) \bmod 1 .
\end{aligned}
$$

From $F_{a, b}^{\circ p}(X)=X+k$, it follows that

$$
\begin{aligned}
\lim _{n \rightarrow \infty} \frac{F_{a, b}^{\circ n p}(X)}{2^{n p}} & =\lim _{n \rightarrow \infty} \frac{X+k \sum_{j=0}^{n-1} 2^{p j}}{2^{n p}} \\
& =\lim _{n \rightarrow \infty} \frac{k \frac{2^{n p}-1}{2^{p}-1}}{2^{n p}} .
\end{aligned}
$$

Proposition 4.3. Let $\tau \in \mathbb{T}^{1}$ be a periodic point of the doubling map D. Then there exists a unique $a \in \mathbb{T}^{1}$ such that $(a, 1)$ is of type $\tau$ and the corresponding cycle of $g_{a, b}$ is superattracting.

Remark 4.4. If $g_{a, b}$ has a superattracting cycle, then $b=1$ (compare section 2).

Proof. As recalled in Remark 1.3] if $\tau \in \mathbb{T}^{1}$ is a periodic point of $D$, then there exist $p \in \mathbb{N}^{*}, k \in\left\{0, \ldots, 2^{p}-2\right\}$ such that $\tau=\frac{k}{2^{p}-1}$.

Moreover, from Lemma 4.1, it follows that for all $p \in \mathbb{N}^{*}$ and all $k \in\left\{0, \ldots, 2^{p}-\right.$ $2\}$ there exists a unique $a \in \mathbb{T}^{1}$ such that $F_{a, b}^{\circ p}(1 / 2)=1 / 2+k$. But then, from Lemma 4.2. the parameter $(a, 1)$ is of type $\tau$.

Corollary 4.5. If $\tau \in \mathbb{T}^{1}$ is a type, the tongue $T_{\tau}$ is non-empty.

Corollary 4.6. The tongues are connected.

Proof. The tongue $T_{\tau}$ is non-empty. Taking any point in this tongue as its starting point, there is a path $\gamma:[0,1] \rightarrow T_{\tau}$ as above. The limit $\gamma(1)$ of this path is a parameter $(a, 1)$ of type $\tau$ with a superattracting cycle, and from the above there is only one such parameter.

\section{Open QUeSTIONS}

The other question left unanswered in reference 5 concerns the order of contact of the left and right boundaries of the tongue. The order of contact might be $\frac{1}{2}$ for all of the tongues. See this reference for a study of examples. We have reason to believe that the order of contact is the same for all of the tongues.

When we consider the dynamics of the family $g_{a, b}$ instead of $f_{a, b}$, we see that there can also be a symmetric pair of attracting cycles in $\mathbb{C}^{*}$. We might expect the corresponding parameters to form Mandelbrot-like families or even tricorn shapes in the parameter space $\mathbb{T}^{1} \times[0,1]$. One can see shapes looking like Mandelbrot sets (see Figure 1) attached on each tongue, with similar non-local connectedness as previous examples (compare [6], 2]). We should note that the complexified double standard family may be related to a rational Blaschke product with the same kind of dynamics near the circle (the restriction to the circle is of degree two and strictly monotonic); see 3 . 

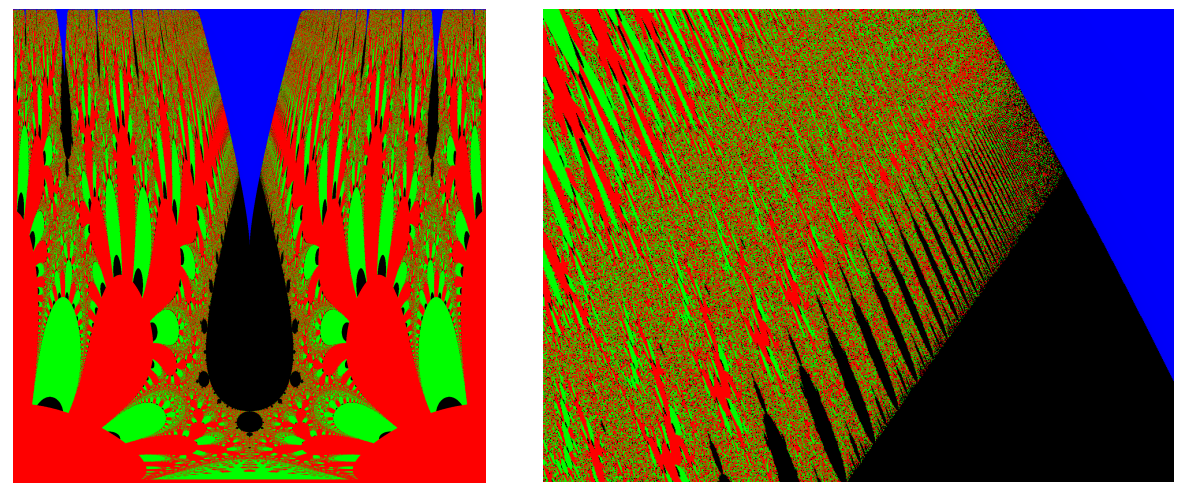

Figure 1. The parameter plane for the family $\left(g_{a, b}\right)_{a, b}$ tongues are dark grey (blue in the electronic version); the parameters with escaping critical points correspond to the lighter greys (red and green in the electronic version); and the others, the parameters for which the critical point stays in a neighbourhood of the circle without tending to it after some iterations, are black. On the right is a magnification of the contact between the Mandelbrot-like shape and the tongue.

\section{ACKNOWLEDGEMENTS}

The author wishes to thank Kuntal Banerjee, Xavier Buff, Adam Epstein, Núria Fagella and the referee.

\section{REFERENCES}

[1] Lars V. Ahlfors. Lectures on quasiconformal mappings, volume 38 of University Lecture Series. American Mathematical Society, Providence, RI, second edition, 2006. With supplemental chapters by C. J. Earle, I. Kra, M. Shishikura and J. H. Hubbard. MR2241787 (2009d:30001)

[2] Adam Epstein and Michael Yampolsky. Geography of the cubic connectedness locus: Intertwining surgery. Ann. Sci. École Norm. Sup. (4), 32(2):151-185, 1999. MR 1681808 (2000i:37067)

[3] Núria Fagella and A. Garijo. Blaschke products of degree 4 and cubic polynomials. In preparation.

[4] Michał Misiurewicz and Ana Rodrigues. Double standard maps. Comm. Math. Phys., 273(1):37-65, 2007. MR 2308749(2008b:37067)

[5] Michał Misiurewicz and Ana Rodrigues. On the tip of the tongue. J. Fixed Point Theory Appl., 3(1):131-141, 2008. MR2402913 (2009g:37037)

[6] Shizuo Nakane and Dierk Schleicher. Non-local connectivity of the tricorn and multicorns. In Dynamical systems and chaos, Vol. 1 (Hachioji, 1994), pages 200-203. World Sci. Publ., River Edge, NJ, 1995. MR1479931

[7] Mitsuhiro Shishikura. On the quasiconformal surgery of rational functions. Ann. Sci. École Norm. Sup. (4), 20(1):1-29, 1987. MR892140(88i:58099)

[8] Saeed Zakeri. On Siegel disks of a class of entire maps. To appear in Duke Math. Journal, 2009.

Institut de mathématiques de Toulouse UMR5219, Université de Toulouse, UPS, 118, route de Narbonne, 31062 Toulouse Cedex, France

E-mail address: dezotti@math.univ-toulouse.fr 